\title{
On the solutions and periodicity of some nonlinear systems of difference equations
}

M. M. El-Dessoky

King AbdulAziz University, Faculty of Science, Mathematics Department, P. O. Box 80203, Jeddah 21589, Saudi Arabia.

Department of Mathematics, Faculty of Science, Mansoura University, Mansoura 35516, Egypt.

Communicated by C. Alaca

Abstract

We investigate the expressions of solutions and the periodicity nature of the following system of rational difference equations of order four

$$
\begin{aligned}
x_{n+1} & =\frac{z_{n-3}}{a_{1}+b_{1} z_{n} y_{n-1} x_{n-2} z_{n-3}}, y_{n+1}=\frac{x_{n-3}}{a_{2}+b_{2} x_{n} z_{n-1} y_{n-2} x_{n-3}}, \\
z_{n+1} & =\frac{y_{n-3}}{a_{3}+b_{3} y_{n} x_{n-1} z_{n-2} y_{n-3}}
\end{aligned}
$$

where the initial conditions $x_{-3}, x_{-2}, x_{-1}, x_{0}, y_{-3}, y_{-2}, y_{-1}, y_{0}, z_{-3}, z_{-2}, z_{-1}$ and $z_{0}$ are arbitrary real numbers and $a_{1}, b_{1}, a_{2}, b_{2}, a_{3}, b_{3}$ are integers. (C) 2016 All rights reserved.

Keywords: System of difference equations, recursive sequences, stability, periodic solution, solution of difference equation.

2010 MSC: 39A10, 39A22, 39A23.

\section{Introduction}

Our goal is to obtain a form of the solutions and the periodicity character of the systems of rational difference equations

$$
x_{n+1}=\frac{z_{n-3}}{a_{1}+b_{1} z_{n} y_{n-1} x_{n-2} z_{n-3}}, y_{n+1}=\frac{x_{n-3}}{a_{2}+b_{2} x_{n} z_{n-1} y_{n-2} x_{n-3}},
$$

Email address: dessokym@mans.edu.eg (M. M. El-Dessoky) 


$$
z_{n+1}=\frac{y_{n-3}}{a_{3}+b_{3} y_{n} x_{n-1} z_{n-2} y_{n-3}},
$$

where the initial conditions are arbitrary real numbers. Also, the constants $a_{i}, b_{i}, i=1,2,3$ are integer numbers. Throughout this paper, we assume that $A=x_{-3} y_{-2} z_{-1} x_{0}, B=z_{-3} x_{-2} y_{-1} z_{0}$ and $C=y_{-3} z_{-2} x_{-1} y_{0}$.

In recent years, with the wide application of computers, difference system has become one of the important theoretical bases for computer, information system, ecological balance, engineering control, biological system, economical systems, and so forth. As typical nonlinear difference equations, rational difference equations have become a research hot spot in mathematical modelling. The behavior of solutions of systems of rational difference equations has received extensive attention. Recently a great effort has been made in studying the qualitative analysis of rational difference equations and their systems; see [1]-[39].

Din et al. 9] studied the equilibrium points, local asymptotic stability of an equilibrium point, instability of equilibrium points, periodicity behavior of positive solutions, and global character of an equilibrium point of the system of rational difference equations

$$
x_{n+1}=\frac{\alpha x_{n-3}}{\beta+\gamma y_{n} y_{n-1} y_{n-2} y_{n-3}}, y_{n+1}=\frac{\alpha_{1} y_{n-3}}{\beta_{1}+\gamma_{1} x_{n} x_{n-1} x_{n-2} x_{n-3}} .
$$

Qianhong Zhang et al. [37] obtained the boundedness, persistence, and global asymptotic stability of positive solution of the system of third-order rational difference equations

$$
x_{n+1}=A+\frac{x_{n}}{y_{n-1} y_{n-2}}, y_{n+1}=B+\frac{y_{n}}{x_{n-1} x_{n-2}} .
$$

El-Dessoky et al. [14] got the form of the solution of the following system of rational difference equations

$$
x_{n+1}=\frac{x_{n-1}}{1+y_{n} x_{n-1}}, y_{n+1}=\frac{y_{n-1}}{1+x_{n} y_{n-1}}, z_{n+1}=\frac{z_{n-m}}{x_{n} y_{n}} .
$$

In [29], Papaschinopoulos et al. studied the existence of a unique positive equilibrium, the boundedness, persistence and global attractivity of the positive solutions of the system of the following two difference equations

$$
x_{n+1}=a x_{n}+b y_{n-1} e^{-x_{n}}, \quad y_{n+1}=c y_{n}+d x_{n-1} e^{-y_{n}} .
$$

Hui-li Ma et al [26] investigated the behavior of positive solutions for the following systems of rational difference equations

$$
x_{n+1}=\frac{A}{x_{n} y_{n}^{2}}, y_{n+1}=\frac{B y_{n}}{x_{n-1} y_{n-1}} .
$$

Özban [28] investigated the periodicity of solutions of the system of difference equations

$$
x_{n+1}=\frac{1}{y_{n-k}}, y_{n+1}=\frac{y_{n}}{x_{n-m} y_{n-m-k}} .
$$

Touafek et al. [33] investigated the periodic nature and got the form of the solutions of the following systems of rational difference equations

$$
x_{n+1}=\frac{x_{n-3}}{ \pm 1 \pm x_{n-3} y_{n-1}}, y_{n+1}=\frac{y_{n-3}}{ \pm 1 \pm y_{n-3} x_{n-1}} .
$$

Banyat Sroysang [30] studied the solutions, stability character, and asymptotic behavior of the system of a rational $m$-th order difference equation

$$
x_{n+1}=\frac{x_{n-m+1}}{A+y_{n} y_{n-1} \ldots, y_{n-m+1}}, y_{n+1}=\frac{y_{n-m+1}}{B+x_{n} x_{n-1} \ldots x_{n-m+1}} .
$$


The behavior of positive solutions of the following system

$$
x_{n+1}=\frac{x_{n-1}}{1+x_{n-1} y_{n}}, y_{n+1}=\frac{y_{n-1}}{1+y_{n-1} x_{n}}
$$

was studied by Kurbanli et al. [24].

In the same year, Kurbanli [22] studied the behavior of the solutions of the system of rational difference equations

$$
x_{n+1}=\frac{x_{n-1}}{x_{n-1} y_{n}-1}, y_{n+1}=\frac{y_{n-1}}{y_{n-1} x_{n}-1}, z_{n+1}=\frac{z_{n-1}}{z_{n-1} y_{n}-1} .
$$

Liu et al. 25] studied the behavior of the solutions of the system of rational difference equations

$$
x_{n+1}=\frac{x_{n-1}}{x_{n-1} y_{n}-1}, y_{n+1}=\frac{y_{n-1}}{y_{n-1} x_{n}-1}, z_{n+1}=\frac{1}{z_{n-1} x_{n}} .
$$

In [5] Cinar obtained the periodicity of the positive solutions of the system of rational difference equations

$$
x_{n+1}=\frac{1}{y_{n}}, y_{n+1}=\frac{y_{n}}{x_{n-1} y_{n-1}} .
$$

Moreover, the system of rational difference equations

$$
x_{n+1}=\frac{x_{n}}{c y_{n}+a}, y_{n+1}=\frac{y_{n}}{d x_{n}+b},
$$

was studied by Clark et al. [6, 7].

\section{The First Case $a_{i}=b_{i}=1, i=1,2,3$}

We investigate, in this section, the solutions of the following system of three difference equations

$$
x_{n+1}=\frac{z_{n-3}}{1+z_{n} y_{n-1} x_{n-2} z_{n-3}}, y_{n+1}=\frac{x_{n-3}}{1+x_{n} z_{n-1} y_{n-2} x_{n-3}}, z_{n+1}=\frac{y_{n-3}}{1+y_{n} x_{n-1} z_{n-2} y_{n-3}},
$$

where $n \in \mathbb{N}_{0}$ and the initial conditions are arbitrary real numbers.

Theorem 2.1. Assume that $\left\{x_{n}, y_{n}, z_{n}\right\}$ are solutions of system 2.1). Then for $n=0,1,2, \ldots$, all the solutions of system (2.1) are given by the following formulae

$$
\begin{aligned}
x_{12 n-3} & =x_{-3} \prod_{i=0}^{n-1} \frac{(1+(12 i) A)(1+(12 i+4) A)(1+(12 i+8) A)}{(1+(12 i+1) A)(1+(12 i+5) A)(1+(12 i+9) A)}, \\
x_{12 n-2} & =x_{-2} \prod_{i=0}^{n-1} \frac{(1+(12 i+1) B)(1+(12 i+5) B)(1+(12 i+9) B)}{(1+(12 i+2) B)(1+(12 i+6) B)(1+(12 i+10) B)}, \\
x_{12 n-1} & =x_{-1} \prod_{i=0}^{n-1} \frac{(1+(12 i+2) C)(1+(12 i+6) C)(1+(12 i+10) C)}{(1+(12 i+3) C)(1+(12 i+7) C)(1+(12 i+11) C)}, \\
x_{12 n} & =x_{0} \prod_{i=0}^{n-1} \frac{(1+(12 i+3) A)(1+(12 i+7) A)(1+(12 i+11) A)}{(1+(12 i+4) A)(1+(12 i+8) A)(1+(12 i+12) A)}, \\
x_{12 n+1} & =\frac{z_{-3}}{1+B} \prod_{i=0}^{n-1} \frac{(1+(12 i+4) B)(1+(12 i+8) B)(1+(12 i+12) B)}{(1+(12 i+5) B)(1+(12 i+9) B)(1+(12 i+13) B)}, \\
x_{12 n+2} & =\frac{z_{-2}(1+C)}{(1+2 C)} \prod_{i=0}^{n-1} \frac{(1+(12 i+5) C)(1+(12 i+9) C)(1+(12 i+13) C)}{(1+(12 i+6) C)(1+(12 i+10) C)(1+(12 i+14) C)},
\end{aligned}
$$




$$
\begin{aligned}
& x_{12 n+3}=\frac{z_{-1}(1+2 A)}{(1+3 A)} \prod_{i=0}^{n-1} \frac{(1+(12 i+6) A)(1+(12 i+10) A)(1+(12 i+14) A)}{(1+(12 i+7) A)(1+(12 i+11) A)(1+(12 i+15) A)}, \\
& x_{12 n+4}=\frac{z_{0}(1+3 B)}{(1+4 B)} \prod_{i=0}^{n-1} \frac{(1+(12 i+7) B)(1+(12 i+11) B)(1+(12 i+15) B)}{(1+(12 i+8) B)(1+(12 i+12) B)(1+(12 i+16) B)}, \\
& x_{12 n+5}=\frac{y_{-3}(1+4 C)}{(1+C)(1+5 C)} \prod_{i=0}^{n-1} \frac{(1+(12 i+8) C)(1+(12 i+12) C)(1+(12 i+16) C)}{(1+(12 i+9) C)(1+(12 i+13) C)(1+(12 i+17) C)} \text {, } \\
& x_{12 n+6}=\frac{y_{-2}(1+A)(1+5 A)}{(1+2 A)(1+6 A)} \prod_{i=0}^{n-1} \frac{(1+(12 i+9) A)(1+(12 i+13) A)(1+(12 i+17) A)}{(1+(12 i+10) A)(1+(12 i+14) A)(1+(12 i+18) A)}, \\
& x_{12 n+7}=\frac{y_{-1}(1+2 B)(1+6 B)}{(1+3 B)(1+7 B)} \prod_{i=0}^{n-1} \frac{(1+(12 i+10) B)(1+(12 i+14) B)(1+(12 i+18) B)}{(1+(12 i+11) B)(1+(12 i+15) B)(1+(12 i+19) B)}, \\
& x_{12 n+8}=\frac{y_{0}(1+3 C)(1+7 C)}{(1+4 C)(1+8 C)} \prod_{i=0}^{n-1} \frac{(1+(12 i+11) C)(1+(12 i+15) C)(1+(12 i+19) C)}{(1+(12 i+12) C)(1+(12 i+16) C)(1+(12 i+20) C)} \text {, } \\
& y_{12 n-3}=y_{-3} \prod_{i=0}^{n-1} \frac{(1+(12 i) C)(1+(12 i+4) C)(1+(12 i+8) C)}{(1+(12 i+1) C)(1+(12 i+5) C)(1+(12 i+9) C)} \text {, } \\
& y_{12 n-2}=y_{-2} \prod_{i=0}^{n-1} \frac{(1+(12 i+1) A)(1+(12 i+5) A)(1+(12 i+9) A)}{(1+(12 i+2) A)(1+(12 i+6) A)(1+(12 i+10) A)}, \\
& y_{12 n-1}=y_{-1} \prod_{i=0}^{n-1} \frac{(1+(12 i+2) B)(1+(12 i+6) B)(1+(12 i+10) B)}{(1+(12 i+3) B)(1+(12 i+7) B)(1+(12 i+11) B)}, \\
& y_{12 n}=y_{0} \prod_{i=0}^{n-1} \frac{(1+(12 i+3) C)(1+(12 i+7) C)(1+(12 i+11) C)}{(1+(12 i+4) C)(1+(12 i+8) C)(1+(12 i+12) C)} \text {, } \\
& y_{12 n+1}=\frac{x_{-3}}{1+A} \prod_{i=0}^{n-1} \frac{(1+(12 i+4) A)(1+(12 i+8) A)(1+(12 i+12) A)}{(1+(12 i+5) A)(1+(12 i+9) A)(1+(12 i+13) A)} \\
& y_{12 n+2}=\frac{x_{-2}(1+B)}{(1+2 B)} \prod_{i=0}^{n-1} \frac{(1+(12 i+5) B)(1+(12 i+9) B)(1+(12 i+13) B)}{(1+(12 i+6) B)(1+(12 i+10) B)(1+(12 i+14) B)} \\
& y_{12 n+3}=\frac{x_{-1}(1+2 C)}{(1+3 C)} \prod_{i=0}^{n-1} \frac{(1+(12 i+6) C)(1+(12 i+10) C)(1+(12 i+14) C)}{(1+(12 i+7) C)(1+(12 i+11) C)(1+(12 i+15) C)}, \\
& y_{12 n+4}=\frac{x_{0}(1+3 A)}{(1+4 A)} \prod_{i=0}^{n-1} \frac{(1+(12 i+7) A)(1+(12 i+11) A)(1+(12 i+15) A)}{(1+(12 i+8) A)(1+(12 i+12) A)(1+(12 i+16) A)}, \\
& y_{12 n+5}=\frac{z_{-3}(1+4 B)}{(1+B)(1+5 B)} \prod_{i=0}^{n-1} \frac{(1+(12 i+8) B)(1+(12 i+12) B)(1+(12 i+16) B)}{(1+(12 i+9) B)(1+(12 i+13) B)(1+(12 i+17) B)}, \\
& y_{12 n+6}=\frac{z_{-2}(1+C)(1+5 C)}{(1+2 C)(1+6 C)} \prod_{i=0}^{n-1} \frac{(1+(12 i+9) C)(1+(12 i+13) C)(1+(12 i+17) C)}{(1+(12 i+10) C)(1+(12 i+14) C)(1+(12 i+18) C)} \text {, } \\
& y_{12 n+7}=\frac{z_{-1}(1+2 A)(1+6 A)}{(1+3 A)(1+7 A)} \prod_{i=0}^{n-1} \frac{(1+(12 i+10) A)(1+(12 i+14) A)(1+(12 i+18) A)}{(1+(12 i+11) A)(1+(12 i+15) A)(1+(12 i+19) A)}, \\
& y_{12 n+8}=\frac{z_{0}(1+3 B)(1+7 B)}{(1+4 B)(1+8 B)} \prod_{i=0}^{n-1} \frac{(1+(12 i+11) B)(1+(12 i+15) B)(1+(12 i+19) B)}{(1+(12 i+12) B)(1+(12 i+16) B)(1+(12 i+20) B)},
\end{aligned}
$$




$$
\begin{aligned}
& z_{12 n-3}=z_{-3} \prod_{i=0}^{n-1} \frac{(1+(12 i) B)(1+(12 i+4) B)(1+(12 i+8) B)}{(1+(12 i+1) B)(1+(12 i+5) B)(1+(12 i+9) B)} \\
& z_{12 n-2}=z_{-2} \prod_{i=0}^{n-1} \frac{(1+(12 i+1) C)(1+(12 i+5) C)(1+(12 i+9) C)}{(1+(12 i+2) C)(1+(12 i+6) C)(1+(12 i+10) C)} \\
& z_{12 n-1}=z_{-1} \prod_{i=0}^{n-1} \frac{(1+(12 i+2) A)(1+(12 i+6) A)(1+(12 i+10) A)}{(1+(12 i+3) A)(1+(12 i+7) A)(1+(12 i+11) A)} \\
& z_{12 n}=z_{0} \prod_{i=0}^{n-1} \frac{(1+(12 i+3) B)(1+(12 i+7) B)(1+(12 i+11) B)}{(1+(12 i+4) B)(1+(12 i+8) B)(1+(12 i+12) B)} \\
& z_{12 n+1}=\frac{y_{-3}}{1+C} \prod_{i=0}^{n-1} \frac{(1+(12 i+4) C)(1+(12 i+8) C)(1+(12 i+12) C)}{(1+(12 i+5) C)(1+(12 i+9) C)(1+(12 i+13) C)} \\
& z_{12 n+2}=\frac{y-2(1+A)}{(1+2 A)} \prod_{i=0}^{n-1} \frac{(1+(12 i+5) A)(1+(12 i+9) A)(1+(12 i+13) A)}{(1+(12 i+6) A)(1+(12 i+10) A)(1+(12 i+14) A)} \\
& z_{12 n+3}=\frac{y_{-1}(1+2 B)}{(1+3 B)} \prod_{i=0}^{n-1} \frac{(1+(12 i+6) B)(1+(12 i+10) B)(1+(12 i+14) B)}{(1+(12 i+7) B)(1+(12 i+11) B)(1+(12 i+15) B)}, \\
& z_{12 n+4}=\frac{y_{0}(1+3 C)}{(1+4 C)} \prod_{i=0}^{n-1} \frac{(1+(12 i+7) C)(1+(12 i+11) C)(1+(12 i+15) C)}{(1+(12 i+8) C)(1+(12 i+12) C)(1+(12 i+16) C)} \\
& z_{12 n+5}=\frac{x_{-3}(1+4 A)}{(1+A)(1+5 A)} \prod_{i=0}^{n-1} \frac{(1+(12 i+8) A)(1+(12 i+12) A)(1+(12 i+16) A)}{(1+(12 i+9) A)(1+(12 i+13) A)(1+(12 i+17) A)}, \\
& z_{12 n+6}=\frac{x_{-2}(1+B)(1+5 B)}{(1+2 B)(1+6 B)} \prod_{i=0}^{n-1} \frac{(1+(12 i+9) B)(1+(12 i+13) B)(1+(12 i+17) B)}{(1+(12 i+10) B)(1+(12 i+14) B)(1+(12 i+18) B)} \\
& z_{12 n+7}=\frac{x_{-1}(1+2 C)(1+6 C)}{(1+3 C)(1+7 C)} \prod_{i=0}^{n-1} \frac{(1+(12 i+10) C)(1+(12 i+14) C)(1+(12 i+18) C)}{(1+(12 i+11) C)(1+(12 i+15) C)(1+(12 i+19) C)} \\
& z_{12 n+8}=\frac{x_{0}(1+3 A)(1+7 A)}{(1+4 A)(1+8 A)} \prod_{i=0}^{n-1} \frac{(1+(12 i+11) A)(1+(12 i+15) A)(1+(12 i+19) A)}{(1+(12 i+12) A)(1+(12 i+16) A)(1+(12 i+20) A)}
\end{aligned}
$$

where $\prod_{i=0}^{-1} t_{i}=1$ and $A, B, C \notin\left\{-\frac{1}{n}, n=1,2, \ldots\right\}$.

Proof. For $n=0$, the assertion holds. Now suppose that $n>0$ and that our assertion holds for $n-1$ that is,

$$
\begin{aligned}
& x_{12 n-7}=\frac{y_{-3}(1+4 C)}{(1+C)(1+5 C)} \prod_{i=0}^{n-2} \frac{(1+(12 i+8) C)(1+(12 i+12) C)(1+(12 i+16) C)}{(1+(12 i+9) C)(1+(12 i+13) C)(1+(12 i+17) C)}, \\
& x_{12 n-6}=\frac{y_{-2}(1+A)(1+5 A)}{(1+2 A)(1+6 A)} \prod_{i=0}^{n-2} \frac{(1+(12 i+9) A)(1+(12 i+13) A)(1+(12 i+17) A)}{(1+(12 i+10) A)(1+(12 i+14) A)(1+(12 i+18) A)}, \\
& x_{12 n-5}=\frac{y_{-1}(1+2 B)(1+6 B)}{(1+3 B)(1+7 B)} \prod_{i=0}^{n-2} \frac{(1+(12 i+10) B)(1+(12 i+14) B)(1+(12 i+18) B)}{(1+(12 i+11) B)(1+(12 i+15) B)(1+(12 i+19) B)}, \\
& x_{12 n-4}=\frac{y_{0}(1+3 C)(1+7 C)}{(1+4 C)(1+8 C)} \prod_{i=0}^{n-2} \frac{(1+(12 i+11) C)(1+(12 i+15) C)(1+(12 i+19) C)}{(1+(12 i+12) C)(1+(12 i+16) C)(1+(12 i+20) C)},
\end{aligned}
$$




$$
\begin{aligned}
& y_{12 n-7}=\frac{z_{-3}(1+4 B)}{(1+B)(1+5 B)} \prod_{i=0}^{n-2} \frac{(1+(12 i+8) B)(1+(12 i+12) B)(1+(12 i+16) B)}{(1+(12 i+9) B)(1+(12 i+13) B)(1+(12 i+17) B)} \\
& y_{12 n-6}=\frac{z_{-2}(1+C)(1+5 C)}{(1+2 C)(1+6 C)} \prod_{i=0}^{n-2} \frac{(1+(12 i+9) C)(1+(12 i+13) C)(1+(12 i+17) C)}{(1+(12 i+10) C)(1+(12 i+14) C)(1+(12 i+18) C)} \\
& y_{12 n-5}=\frac{z_{-1}(1+2 A)(1+6 A)}{(1+3 A)(1+7 A)} \prod_{i=0}^{n-2} \frac{(1+(12 i+10) A)(1+(12 i+14) A)(1+(12 i+18) A)}{(1+(12 i+11) A)(1+(12 i+15) A)(1+(12 i+19) A)} \\
& y_{12 n-4}=\frac{z_{0}(1+3 B)(1+7 B)}{(1+4 B)(1+8 B)} \prod_{i=0}^{n-2} \frac{(1+(12 i+11) B)(1+(12 i+15) B)(1+(12 i+19) B)}{(1+(12 i+12) B)(1+(12 i+16) B)(1+(12 i+20) B)} \\
& z_{12 n-7}=\frac{x_{-3}(1+4 A)}{(1+A)(1+5 A)} \prod_{i=0}^{n-2} \frac{(1+(12 i+8) A)(1+(12 i+12) A)(1+(12 i+16) A)}{(1+(12 i+9) A)(1+(12 i+13) A)(1+(12 i+17) A)} \\
& z_{12 n-6}=\frac{x_{-2}(1+B)(1+5 B)}{(1+2 B)(1+6 B)} \prod_{i=0}^{n-2} \frac{(1+(12 i+9) B)(1+(12 i+13) B)(1+(12 i+17) B)}{(1+(12 i+10) B)(1+(12 i+14) B)(1+(12 i+18) B)} \\
& z_{12 n-5}=\frac{x_{-1}(1+2 C)(1+6 C)}{(1+3 C)(1+7 C)} \prod_{i=0}^{n-2} \frac{(1+(12 i+10) C)(1+(12 i+14) C)(1+(12 i+18) C)}{(1+(12 i+11) C)(1+(12 i+15) C)(1+(12 i+19) C)} \\
& z_{12 n-4}=\frac{x_{0}(1+3 A)(1+7 A)}{(1+4 A)(1+8 A)} \prod_{i=0}^{n-2} \frac{(1+(12 i+11) A)(1+(12 i+15) A)(1+(12 i+19) A)}{(1+(12 i+12) A)(1+(12 i+16) A)(1+(12 i+20) A)}
\end{aligned}
$$

From 2.1), we see that

$$
\begin{aligned}
& x_{12 n-3}=\frac{z_{12 n-7}}{1+z_{12 n-4} y_{12 n-5} x_{12 n-6} z_{12 n-7}} \\
& =\frac{\frac{x_{-3}(1+4 A)}{(1+A)(1+5 A)} \prod_{i=0}^{n-2} \frac{(1+(12 i+8) A)(1+(12 i+12) A)(1+(12 i+16) A)}{(1+(12 i+9) A)(1+(12 i+13) A)(1+(12 i+17) A)}}{\left(\begin{array}{c}
1+\frac{x_{0}(1+3 A)(1+7 A)}{(1+4 A)(1+8 A)} \prod_{i=0}^{n-2} \frac{(1+(12 i+11) A)(1+(12 i+15) A)(1+(12 i+19) A)}{(1+(12 i+12) A)(1+(12 i+16) A)(1+(12 i+20) A)} \\
\frac{z_{-1}(1+2 A)(1+6 A)}{(1+3 A)(1+7 A)} \prod_{i=0}^{n-2} \frac{(1+(12 i+10) A)(1+(12 i+14) A)(1+(12 i+18) A)}{(1+(12 i+11) A)(1+(12 i+15) A)(1+(12 i+19) A)} \\
\frac{y_{-2}(1+A)(1+5 A)}{(1+2 A)(1+6 A)} \prod_{i=0}^{n-2} \frac{(1+(12 i+9) A)(1+(12 i+13) A)(1+(12 i+17) A)}{(1+(12 i+10) A)(1+(12 i+14) A)(1+(12 i+18) A)} \\
\frac{x_{-3}(1+4 A)}{(1+A)(1+5 A)} \prod_{i=0}^{n-2} \frac{(1+(12 i+8) A)(1+(12 i+12) A)(1+(12 i+16) A)}{(1+(12 i+9) A)(1+(12 i+13) A)(1+(12 i+17) A)}
\end{array}\right)} \\
& \frac{x_{-3}(1+4 A)}{(1+A)(1+5 A)} \prod_{i=0}^{n-2} \frac{(1+(12 i+8) A)(1+(12 i+12) A)(1+(12 i+16) A)}{(1+(12 i+9) A)(1+(12 i+13) A)(1+(12 i+17) A)} \\
& =\frac{\left(1+\frac{A}{(1+8 A)} \prod_{i=0}^{n-2} \frac{(1+(12 i+8) A)}{(1+(12 i+20) A)}\right)}{(1=0} \\
& =\frac{x_{-3}(1+4 A)}{(1+A)(1+5 A)\left(1+\frac{A}{(1+(12 n-4) A)}\right)} \prod_{i=0}^{n-2} \frac{(1+(12 i+8) A)(1+(12 i+12) A)(1+(12 i+16) A)}{(1+(12 i+9) A)(1+(12 i+13) A)(1+(12 i+17) A)} \\
& =\frac{x_{-3}(1+4 A)(1+(12 n-4) A)}{(1+A)(1+5 A)(1+(12 n-3) A)} \prod_{i=0}^{n-2} \frac{(1+(12 i+8) A)(1+(12 i+12) A)(1+(12 i+16) A)}{(1+(12 i+9) A)(1+(12 i+13) A)(1+(12 i+17) A)} \\
& =x_{-3} \prod_{i=0}^{n-1} \frac{(1+(12 i) A)(1+(12 i+4) A)(1+(12 i+8) A)}{(1+(12 i+1) A)(1+(12 i+5) A)(1+(12 i+9) A)} .
\end{aligned}
$$




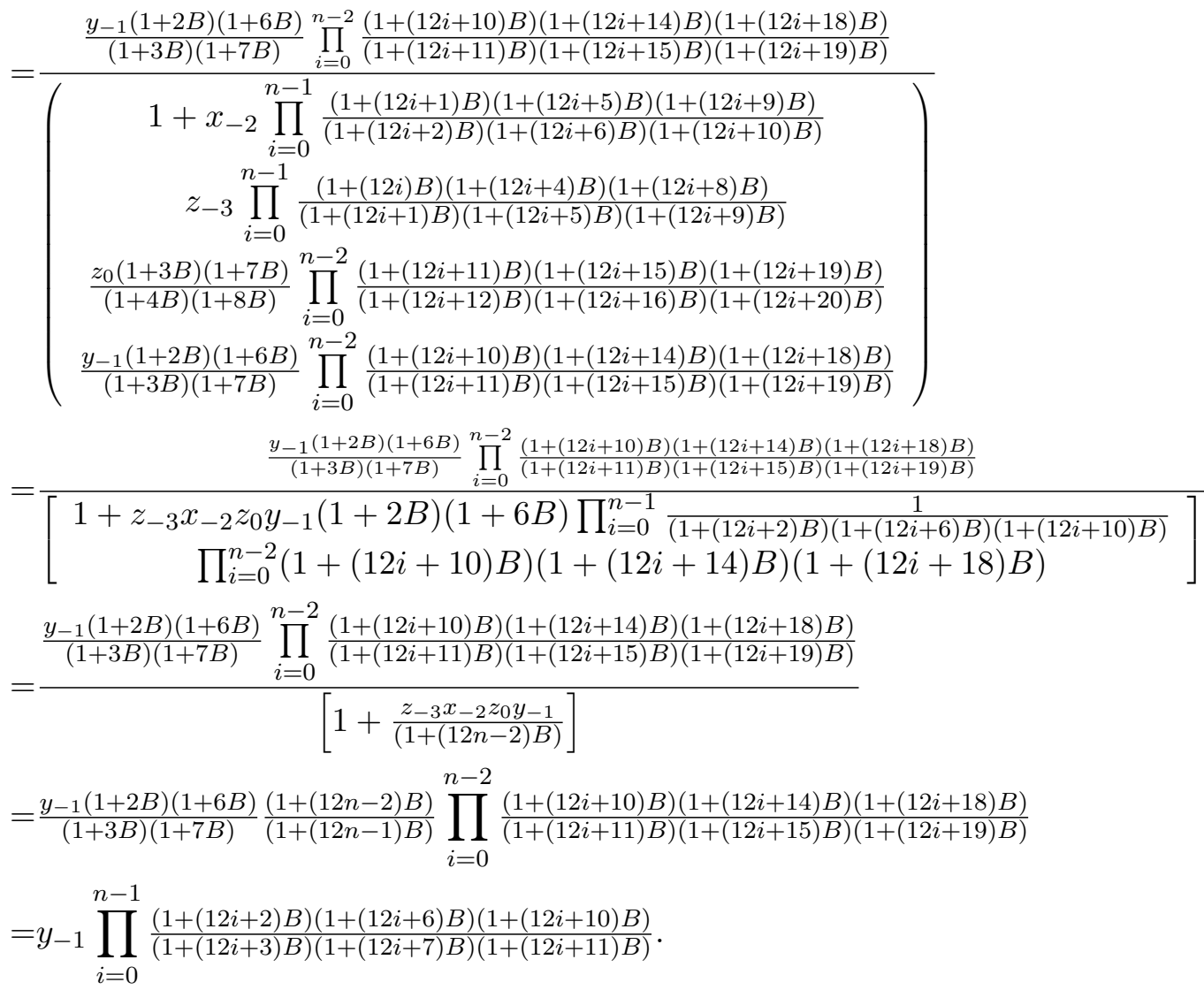

Also, we see from (2.1) that

$$
\begin{aligned}
& z_{12 n}=\frac{y_{12 n-4}}{1+y_{12 n-1} x_{12 n-2} z_{12 n-3} y_{12 n-4}} \\
& =\frac{\left(\frac{z_{0}(1+3 B)(1+7 B)}{(1+4 B)(1+8 B)} \prod_{i=0}^{n-2} \frac{(1+(12 i+11) B)(1+(12 i+15) B)(1+(12 i+19) B)}{(1+(12 i+12) B)(1+(12 i+16) B)(1+(12 i+20) B)}\right)}{\left[\begin{array}{c}
1+\left(y_{-1} \prod_{i=0}^{n-1} \frac{(1+(12 i+2) B)(1+(12 i+6) B)(1+(12 i+10) B)}{(1+(12 i+3) B)(1+(12 i+7) B)(1+(12 i+11) B)}\right) \\
\left(x_{-}-\prod_{i=0}^{n-1} \frac{(1+(12 i+1) B)(1+(12 i+5) B)(1+(12 i+9) B)}{(1+(12 i+2) B)(1+(12 i+6) B)(1+(12 i+10) B)}\right) \\
\left(z_{-3} \prod_{i=0}^{n-1} \frac{(1+(12 i) B)(1+(12 i+4) B)(1+(12 i+8) B)}{(1+(12 i+1) B)(1+(12 i+5) B)(1+(12 i+9) B)}\right) \\
\left(\frac{z_{0}(1+3 B)(1+7 B)}{(1+4 B)(1+8 B)} \prod_{i=0}^{n-2} \frac{(1+(12 i+11) B)(1+(12 i+15) B)(1+(12 i+19) B)}{(1+(12 i+12) B)(1+(12 i+16) B)(1+(12 i+20) B)}\right)
\end{array}\right]} \\
& =\frac{\left(\frac{z_{0}(1+3 B)(1+7 B)}{(1+4 B)(1+8 B)} \prod_{i=0}^{n-2} \frac{(1+(12 i+11) B)(1+(12 i+15) B)(1+(12 i+19) B)}{(1+(12 i+12) B)(1+(12 i+16) B)(1+(12 i+20) B)}\right)}{\left[1+z_{0} y_{-1} x_{-2} z_{-3} \prod_{i=0}^{n-1} \frac{1}{(1+(12 i+11) B)} \prod_{i=0}^{n-2}(1+(12 i+11) B)\right]} \\
& =\frac{\left(\frac{z_{0}(1+3 B)(1+7 B)}{(1+4 B)(1+8 B)} \prod_{i=0}^{n-2} \frac{(1+(12 i+11) B)(1+(12 i+15) B)(1+(12 i+19) B)}{(1+(12 i+12) B)(1+(12 i+16) B)(1+(12 i+20) B)}\right)}{\left[1+\frac{B}{(1+(12 n-1) B)}\right]} \\
& z_{12 n}=\frac{z_{0}(1+3 B)(1+7 B)(1+(12 n-1) B)}{(1+4 B)(1+8 B)(1+(12 n) B)} \prod_{i=0}^{n-2} \frac{(1+(12 i+11) B)(1+(12 i+15) B)(1+(12 i+19) B)}{(1+(12 i+12) B)(1+(12 i+16) B)(1+(12 i+20) B)}
\end{aligned}
$$




$$
=z_{0} \prod_{i=0}^{n-1} \frac{(1+(12 i+3) B)(1+(12 i+7) B)(1+(12 i+11) B)}{(1+(12 i+4) B)(1+(12 i+8) B)(1+(12 i+12) B)} .
$$

Also, we can prove the other relations. This completes the proof.

Lemma 2.2. Let $\left\{x_{n}, y_{n}, z_{n}\right\}$ be a positive solution of system (2.1); then $\left\{x_{n}\right\},\left\{y_{n}\right\}$, and $\left\{z_{n}\right\}$ are bounded and converge to zero.

Proof. It follows from 2.1 that

$$
\begin{aligned}
x_{n+1} & =\frac{z_{n-3}}{1+z_{n} y_{n-1} x_{n-2} z_{n-3}}<z_{n-3}, y_{n+1}=\frac{x_{n-3}}{1+x_{n} z_{n-1} y_{n-2} x_{n-3}}<x_{n-3}, \\
z_{n+1} & =\frac{y_{n-3}}{1+y_{n} x_{n-1} z_{n-2} y_{n-3}}<y_{n-3} .
\end{aligned}
$$

Thus

$$
\begin{gathered}
x_{n+5}<z_{n+1}, y_{n+5}<x_{n+1}, \quad z_{n+5}<y_{n+1} \Rightarrow x_{n+5}<y_{n-3}, y_{n+5}<z_{n-3}, z_{n+5}<x_{n-3} \\
\Rightarrow x_{n+9}<y_{n+1}<x_{n-3}, y_{n+9}<z_{n+1}<y_{n-3}, \quad z_{n+9}<x_{n+1}<z_{n-3} .
\end{gathered}
$$

Then the subsequences $\left\{x_{12 n+i}\right\}_{n=0}^{\infty}, i=-3,-2,-1,0,1,2, \ldots, 8$, are decreasing and bounded from above by $M=\max \left\{x_{-3}, x_{-2}, x_{-1}, x_{0}, \ldots, x_{8}\right\}$. Also, the subsequences

$$
\left\{y_{12 n+i}\right\}_{n=0}^{\infty} \text { and }\left\{z_{12 n+i}\right\}_{n=0}^{\infty}, i=-3,-2,-1,0,1,2, \ldots, 8
$$

are decreasing and bounded from above by $L=\max \left\{y_{-3}, y_{-2}, \ldots, y_{8}\right\}, N=\max \left\{z_{-3}, z_{-2}, \ldots, z_{8}\right\}$ respectively. This completes the proof.

Lemma 2.3. If $x_{i}, y_{i}, z_{i}, i=-3,-2,-1,0$, are arbitrary real numbers and $\left\{x_{n}, y_{n}, z_{n}\right\}$ a solution of system 2.1], then the following statements are true:

(i) If $x_{-3}=0$, then we have $x_{12 n-3}=y_{12 n+1}=z_{12 n+5}=0$ and $x_{12 n}=y_{12 n+4}=z_{12 n+8}=x_{0}, x_{12 n+6}=$ $y_{12 n-2}=z_{12 n+2}=y_{-2}, x_{12 n+3}=y_{12 n+7}=z_{12 n-1}=z_{-1}$.

(ii) If $x_{-2}=0$, then we have $x_{12 n-2}=y_{12 n+2}=z_{12 n+6}=0$, and $x_{12 n+1}=y_{12 n+5}=z_{12 n-3}=z_{-3}$, $x_{12 n+4}=y_{12 n+8}=z_{12 n}=z_{0}, x_{12 n+7}=y_{12 n-1}=z_{12 n+3}=y_{-1}$.

(iii) If $x_{-1}=0$, then we have $x_{12 n-1}=y_{12 n+3}=z_{12 n+7}=0$, and $x_{12 n+2}=y_{12 n+6}=z_{12 n-2}=z_{-2}$, $x_{12 n+5}=y_{12 n-3}=z_{12 n+1}=y_{-3}, x_{12 n+8}=y_{12 n}=z_{12 n+4}=y_{0}$.

(iv) If $x_{0}=0$, then we have $x_{12 n}=y_{12 n+4}=z_{12 n+8}=0$ and $x_{12 n-3}=y_{12 n+1}=z_{12 n+5}=x_{-3}, x_{12 n+3}=$ $y_{12 n+7}=z_{12 n-1}=z_{-1}, x_{12 n+6}=y_{12 n-2}=z_{12 n+2}=y_{-2}$.

(v) If $y_{-3}=0$, then we have $x_{12 n+5}=y_{12 n-3}=z_{12 n+1}=0$ and $x_{12 n-1}=y_{12 n+3}=z_{12 n+7}=x_{-1}$, $x_{12 n+2}=y_{12 n+6}=z_{12 n-2}=z_{-2}, x_{12 n+8}=y_{12 n}=z_{12 n+4}=y_{0}$.

(vi) If $y_{-2}=0$, then we have $x_{12 n+6}=y_{12 n-2}=z_{12 n+2}=0$ and $x_{12 n-3}=y_{12 n+1}=z_{12 n+5}=x_{-3}$, $x_{12 n}=y_{12 n+4}=z_{12 n+8}=x_{0}, x_{12 n+3}=y_{12 n+7}=z_{12 n-1}=z_{-1}$.

(vii) If $y_{-1}=0$, then we have $x_{12 n+7}=y_{12 n-1}=z_{12 n+3}=0$, and $x_{12 n-2}=y_{12 n+2}=z_{12 n+6}=x_{-2}$, $x_{12 n+1}=y_{12 n+5}=z_{12 n-3}=z_{-3}, x_{12 n+4}=y_{12 n+8}=z_{12 n}=z_{0}$.

(viii) If $y_{0}=0$, then we have $x_{12 n+8}=y_{12 n}=z_{12 n+4}=0$ and $x_{12 n-1}=y_{12 n+3}=z_{12 n+7}=x_{-1}, x_{12 n+2}=$ $y_{12 n+6}=z_{12 n-2}=z_{-2}, x_{12 n+5}=y_{12 n-3}=z_{12 n+1}=y_{-3}$.

(ix) If $z_{-3}=0$, then we have $x_{12 n+1}=y_{12 n+5}=z_{12 n-3}=0$ and $x_{12 n-2}=y_{12 n+2}=z_{12 n+6}=x_{-2}$, $x_{12 n+4}=y_{12 n+8}=z_{12 n}=z_{0}, x_{12 n+7}=y_{12 n-1}=z_{12 n+3}=y_{-1}$. 
(x) If $z_{-2}=0$, then we have $x_{12 n+2}=y_{12 n+6}=z_{12 n-2}=0$ and $x_{12 n-1}=y_{12 n+3}=z_{12 n+7}=x_{-1}$, $x_{12 n+5}=y_{12 n-3}=z_{12 n+1}=y_{-3}, x_{12 n+8}=y_{12 n}=z_{12 n+4}=y_{0}$.

(xi) If $z_{-1}=0$, then we have $x_{12 n+3}=y_{12 n+7}=z_{12 n-1}=0$ and $x_{12 n-3}=y_{12 n+1}=z_{12 n+5}=x_{-3}$, $x_{12 n}=y_{12 n+4}=z_{12 n+8}=x_{0}, x_{12 n+6}=y_{12 n-2}=z_{12 n+2}=y_{-2}$.

(xii) If $z_{0}=0$, then we have $x_{12 n+4}=y_{12 n+8}=z_{12 n}=0$ and $x_{12 n-2}=y_{12 n+2}=z_{12 n+6}=x_{-2}, x_{12 n+1}=$ $y_{12 n+5}=z_{12 n-3}=z_{-3}, x_{12 n+7}=y_{12 n-1}=z_{12 n+3}=y_{-1}$.

Proof. The proof follows from the form of the solutions of system 2.1).

Example 2.4. For confirming the results of this section, we consider a numerical example for the difference system (2.1) with the initial conditions $x_{-3}=0.6, x_{-2}=-0.3, x_{-1}=0.8, x_{0}=-1.3, y_{-3}=0.2, y_{-2}=$ $-4, y_{-1}=0.2, y_{0}=0.7, z_{-3}=0.8, z_{-2}=1.1, z_{-1}=0.02$ and $z_{0}=0.4$. (See Fig. 1).

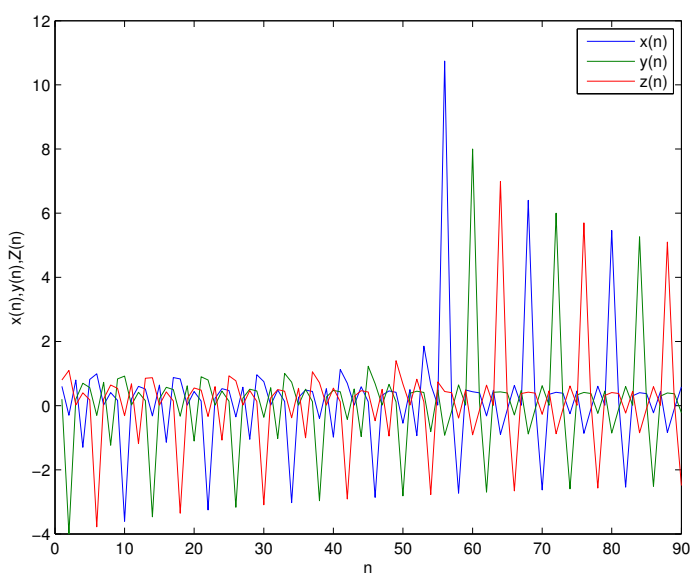

Figure 1: Plot of system 2.1.

Example 2.5. We consider interesting numerical example for the difference system (2.1) with the initial conditions $x_{-3}=0.6, x_{-2}=-0.3, x_{-1}=0, x_{0}=-1.3, y_{-3}=2, y_{-2}=-4, y_{-1}=0.2, y_{0}=0.7, z_{-3}=$ $0.8, z_{-2}=1.1, z_{-1}=0$, and $z_{0}=0.4$ (See Fig. 2).

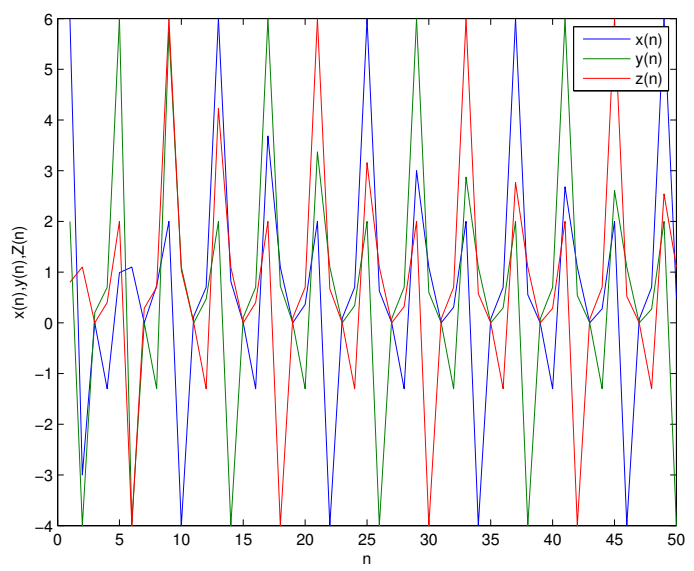

Figure 2: Plot of periodicity of system 2.1. 
3. The Second Case $a_{i}=-1, b_{i}=1, i=1,2,3$

In this section, we obtain the form of the solutions of the system of three difference equations

$$
x_{n+1}=\frac{z_{n-3}}{-1+z_{n} y_{n-1} x_{n-2} z_{n-3}}, y_{n+1}=\frac{x_{n-3}}{-1+x_{n} z_{n-1} y_{n-2} x_{n-3}}, z_{n+1}=\frac{y_{n-3}}{-1+y_{n} x_{n-1} z_{n-2} y_{n-3}},
$$

where $n \in \mathbb{N}_{0}$ and the initial conditions are arbitrary real numbers with $A, B, C \neq 1$.

The following theorem is devoted to the expression of the form of the solutions of system (3.1).

Theorem 3.1. Let $\left\{x_{n}, y_{n}, z_{n}\right\}_{n=-3}^{+\infty}$ be a solution of system (3.1). Then $\left\{x_{n}\right\}_{n=-3}^{+\infty},\left\{y_{n}\right\}_{n=-3}^{+\infty}$ and $\left\{z_{n}\right\}_{n=-3}^{+\infty}$ are given by the formulae, for $n=0,1,2, \ldots$,

$$
\begin{aligned}
x_{12 n-3} & =\frac{x_{-3}}{(-1+A)^{3 n}}, x_{12 n-2}=x_{-2}(-1+B)^{3 n}, x_{12 n-1}=\frac{x_{-1}}{(-1+C)^{3 n}}, \\
x_{12 n} & =x_{0}(-1+A)^{3 n}, x_{12 n+1}=\frac{z_{-3}}{(-1+B)^{3 n+1}}, x_{12 n+2}=z_{-2}(-1+C)^{3 n+1}, \\
x_{12 n+3} & =\frac{z_{-1}}{(-1+A)^{3 n+1}}, x_{12 n+4}=z_{0}(-1+B)^{3 n+1}, x_{12 n+5}=\frac{y_{-3}}{(-1+C)^{3 n+2}}, \\
x_{12 n+6} & =y_{-2}(-1+A)^{3 n+2}, x_{12 n+7}=\frac{y_{-1}}{(-1+B)^{3 n+2}}, x_{12 n+8}=y_{0}(-1+C)^{3 n+2}, \\
y_{12 n-3} & =\frac{y_{-3}}{(-1+C)^{3 n}}, \quad y_{12 n-2}=y_{-2}(-1+A)^{3 n}, y_{12 n-1}=\frac{y_{-1}}{(-1+B)^{3 n}}, \\
y_{12 n} & =y_{0}(-1+C)^{3 n}, y_{12 n+1}=\frac{x_{-3}}{(-1+A)^{3 n+1}}, y_{12 n+2}=x_{-2}(-1+B)^{3 n+1}, \\
y_{12 n+3} & =\frac{x_{-1}}{(-1+C)^{3 n+1}}, y_{12 n+4}=x_{0}(-1+A)^{3 n+1}, y_{12 n+5}=\frac{z_{-3}}{(-1+B)^{3 n+2}}, \\
y_{12 n+6} & =z_{-2}(-1+C)^{3 n+2}, y_{12 n+7}=\frac{z_{-1}}{(-1+A)^{3 n+2}}, y_{12 n+8}=z_{0}(-1+B)^{3 n+2}, \\
z_{12 n-3} & =\frac{z_{-3}}{(-1+B)^{3 n}}, z_{12 n-2}=z_{-2}(-1+C)^{3 n}, z_{12 n-1}=\frac{z_{-1}}{(-1+A)^{3 n}}, \\
z_{12 n} & =z_{0}(-1+B)^{3 n}, z_{12 n+1}=\frac{y_{-3}}{(-1+C)^{3 n+1}}, z_{12 n+2}=y_{-2}(-1+A)^{3 n+1}, \\
z_{12 n+3} & =\frac{y_{-1}}{(-1+B)^{3 n+1}}, z_{12 n+4}=y_{0}(-1+C)^{3 n+1}, z_{12 n+5}=\frac{x_{-3}}{(-1+A)^{3 n+2}}, \\
z_{12 n+6} & =x_{-2}(-1+B)^{3 n+2}, z_{12 n+7}=\frac{x_{-1}}{(-1+C)^{3 n+2}}, z_{12 n+8}=x_{0}(-1+A)^{3 n+2} .
\end{aligned}
$$

Proof. For $n=0$ the assertion holds. Now suppose that $n>0$ and that our assertion holds for $n-1$, that is,

$$
\begin{aligned}
& x_{12 n-7}=\frac{y_{-3}}{(-1+C)^{3 n-1}}, x_{12 n-6}=y_{-2}(-1+A)^{3 n-1}, \\
& x_{12 n-5}=\frac{y_{-1}}{(-1+B)^{3 n-1}}, x_{12 n-4}=y_{0}(-1+C)^{3 n-1}, \\
& y_{12 n-7}=\frac{z_{-3}}{(-1+B)^{3 n-1}}, y_{12 n-6}=z_{-2}(-1+C)^{3 n-1}, \\
& y_{12 n-5}=\frac{z_{-1}}{(-1+A)^{3 n-1}}, y_{12 n-4}=z_{0}(-1+B)^{3 n-1}, \\
& z_{12 n-7}=\frac{x_{-3}}{(-1+A)^{3 n-1}}, \quad z_{12 n-6}=x_{-2}(-1+B)^{3 n-1}, \\
& z_{12 n-5}=\frac{x_{-1}}{(-1+C)^{3 n-1}}, z_{12 n-4}=x_{0}(-1+A)^{3 n-1} .
\end{aligned}
$$


Now it follows from 3.1 that

$$
\begin{aligned}
x_{12 n-3}= & \frac{z_{12 n-7}}{-1+z_{12 n-4} y_{12 n-5} x_{12 n-6} z_{12 n-7}} \\
= & \frac{\left(\frac{x_{-3}}{(-1+A)^{3 n-1}}\right)}{-1+\left(\left(x_{0}(-1+A)^{3 n-1}\right)\left(\frac{z_{-1}}{(-1+A)^{3 n-1}}\right)\left(y_{-2}(-1+A)^{3 n-1}\right)\left(\frac{x_{-3}}{(-1+A)^{3 n-1}}\right)\right)} \\
= & \frac{\left(\frac{x_{-3}}{(-1+A)^{3 n-1}}\right)}{-1+A}=\frac{x_{-3}}{(-1+A)^{3 n}} . \\
y_{12 n-2}= & \frac{x_{12 n-6}}{-1+x_{12 n-3} z_{12 n-4} y_{12 n-5} x_{12 n-6}} \\
= & \frac{\left(y_{-2}(-1+A)^{3 n-1}\right)}{-1+\left(\left(\frac{x_{-3}}{(-1+A)^{3 n}}\right)\left(x_{0}(-1+A)^{3 n-1}\right)\left(\frac{z_{-1}}{(-1+A)^{3 n-1}}\right)\left(y_{-2}(-1+A)^{3 n-1}\right)\right)} \\
= & \frac{\left(y_{-2}(-1+A)^{3 n-1}\right)}{-1+\left(\frac{A}{(-1+A)}\right)}=y_{-2}(-1+A)^{3 n} . \\
z_{12 n}= & \frac{y_{12 n-4}}{-1+y_{12 n-1} x_{12 n-2} z_{12 n-3} y_{12 n-4}} \\
= & \frac{\left(z_{0}(-1+B)^{3 n-1}\right)}{-1+\left(\left(\frac{y_{-1}}{(-1+B)^{3 n}}\right)\left(x_{-2}(-1+B)^{3 n}\right)\left(\frac{z_{-3}}{(-1+B)^{3 n}}\right)\left(z_{0}(-1+B)^{3 n-1}\right)\right)} \\
= & \frac{z_{0}(-1+B)^{3 n-1}}{-1+\frac{B}{(-1+B)}}=z_{0}(-1+B)^{3 n}
\end{aligned}
$$

wherefrom

$$
\begin{aligned}
x_{12 n+1} & =\frac{z_{12 n-3}}{-1+z_{12 n} y_{12 n-1} x_{12 n-2} z_{12 n-3}} \\
& =\frac{\left(\frac{z_{-3}}{(-1+B)^{3 n}}\right)}{-1+\left(\left(z_{0}(-1+B)^{3 n}\right)\left(\frac{y_{-1}}{(-1+B)^{3 n}}\right)\left(x_{-2}(-1+B)^{3 n}\right)\left(\frac{z_{-3}}{(-1+B)^{3 n}}\right)\right)} \\
& =\frac{\left(\frac{z_{-3}}{(-1+B)^{3 n}}\right)}{-1+B}=\frac{z_{-3}}{(-1+B)^{3 n+1}} . \\
y_{12 n+3} & =\frac{x_{12 n-1}}{-1+x_{12 n+2} z_{12 n+1} y_{12 n} x_{12 n-1}}\left(\frac{x_{-1}}{(-1+C)^{3 n}}\right) \\
& =\frac{y_{-3}}{-1+\left(\left(z_{-2}(-1+C)^{3 n+1}\right)\left(\frac{x_{-1}}{(-1+C)^{3 n+1}}\right)\left(y_{0}(-1+C)^{3 n}\right)\left(\frac{x_{-1}}{(-1+C)^{3 n}}\right)\right)} \\
& =\frac{\left(\frac{x_{-1}}{(-1+C)^{3 n}}\right)}{-1+C}=\frac{y_{12 n}}{(-1+C)^{3 n+1}} . \\
z_{12 n+4}= & \frac{\left(y_{0}(-1+C)^{3 n}\right)}{-1+y_{12 n+3} x_{12 n+2} z_{12 n+1} y_{12 n}} \\
= & \frac{\left.x_{-1} z_{-2}(-1+C)^{3 n+1} \frac{y_{-3}}{(-1+C)^{3 n+1}} y_{0}(-1+C)^{3 n}\right)}{-1+\left(\frac{C}{(-1+C)^{3 n+1}} z\right.} \\
= & \frac{y_{0}(-1+C)^{3 n}}{-1+\left(\frac{C}{(-1+C)}\right)}=y_{0}(-1+C)^{3 n+1} .
\end{aligned}
$$

Also, we can prove the other relations. This completes the proof.

Lemma 3.2. The solutions of system (3.1) are unbounded except in the cases from the following two theorems. 
Theorem 3.3. System (3.1) has a periodic solution of period twelve iff $A=B=C=2$ and it can be written in the following form

$$
\begin{aligned}
& \left\{x_{n}\right\}=\left\{x_{-3}, x_{-2}, x_{-1}, x_{0}, z_{-3}, z_{-2}, z_{-1}, z_{0}, y_{-3}, y_{-2}, y_{-1}, y_{0}, x_{-3}, x_{-2}, x_{-1}, x_{0}, \ldots\right\}, \\
& \left\{y_{n}\right\}=\left\{y_{-3}, y_{-2}, y_{-1}, y_{0}, x_{-3}, x_{-2}, x_{-1}, x_{0}, z_{-3}, z_{-2}, z_{-1}, z_{0}, y_{-3}, y_{-2}, y_{-1}, y_{0}, \ldots\right\} \\
& \left\{z_{n}\right\}=\left\{z_{-3}, z_{-2}, z_{-1}, z_{0}, y_{-3}, y_{-2}, y_{-1}, y_{0}, x_{-3}, x_{-2}, x_{-1}, x_{0}, z_{-3}, z_{-2}, z_{-1}, z_{0}, \ldots\right\}
\end{aligned}
$$

Proof. First suppose that there exists a periodic solution of period twelve

$$
\begin{aligned}
& \left\{x_{n}\right\}=\left\{x_{-3}, x_{-2}, x_{-1}, x_{0}, z_{-3}, z_{-2}, z_{-1}, z_{0}, y_{-3}, y_{-2}, y_{-1}, y_{0}, x_{-3}, x_{-2}, x_{-1}, x_{0}, \ldots\right\}, \\
& \left\{y_{n}\right\}=\left\{y_{-3}, y_{-2}, y_{-1}, y_{0}, x_{-3}, x_{-2}, x_{-1}, x_{0}, z_{-3}, z_{-2}, z_{-1}, z_{0}, y_{-3}, y_{-2}, y_{-1}, y_{0}, \ldots\right\}, \\
& \left\{z_{n}\right\}=\left\{z_{-3}, z_{-2}, z_{-1}, z_{0}, y_{-3}, y_{-2}, y_{-1}, y_{0}, x_{-3}, x_{-2}, x_{-1}, x_{0}, z_{-3}, z_{-2}, z_{-1}, z_{0}, \ldots\right\}
\end{aligned}
$$

of system 3.1. From its form, we see that

$$
\begin{aligned}
& x_{-3}=\frac{x_{-3}}{(-1+A)^{3 n}}, x_{-2}=x_{-2}(-1+B)^{3 n}, x_{-1}=\frac{x_{-1}}{(-1+C)^{3 n}}, x_{0}=x_{0}(-1+A)^{3 n}, \\
& z_{-3}=\frac{z_{-3}}{(-1+B)^{3 n+1}}, z_{-2}=z_{-2}(-1+C)^{3 n+1}, z_{-1}=\frac{z_{-1}}{(-1+A)^{3 n+1}}, z_{0}=z_{0}(-1+B)^{3 n+1}, \\
& y_{-3}=\frac{y_{-3}}{(-1+C)^{3 n+2}}, y_{-2}=y_{-2}(-1+A)^{3 n+2}, y_{-1}=\frac{y_{-1}}{(-1+B)^{3 n+2}}, y_{0}=y_{0}(-1+C)^{3 n+2}, \\
& y_{-3}=\frac{y_{-3}}{(-1+C)^{3 n}}, \quad y_{-2}=y_{-2}(-1+A)^{3 n}, y_{-1}=\frac{y_{-1}}{(-1+B)^{3 n}}, y_{0}=y_{0}(-1+C)^{3 n}, \\
& x_{-3}=\frac{x_{-3}}{(-1+A)^{3 n+1}}, x_{-2}=x_{-2}(-1+B)^{3 n+1}, x_{-1}=\frac{x_{-1}}{(-1+C)^{3 n+1}}, x_{0}=x_{0}(-1+A)^{3 n+1}, \\
& z_{-3}=\frac{z_{-3}}{(-1+B)^{3 n+2}}, z_{-2}=z_{-2}(-1+C)^{3 n+2}, z_{-1}=\frac{z_{-1}}{(-1+A)^{3 n+2}}, z_{0}=z_{0}(-1+B)^{3 n+2}, \\
& z_{-3}=\frac{z_{-3}}{(-1+B)^{3 n}}, z_{-2}=z_{-2}(-1+C)^{3 n}, z_{-1}=\frac{z_{-1}}{(-1+A)^{3 n}}, z_{0}=z_{0}(-1+B)^{3 n}, \\
& y_{-3}=\frac{y_{-3}}{(-1+C)^{3 n+1}}, y_{-2}=y_{-2}(-1+A)^{3 n+1}, y_{-1}=\frac{y_{-1}}{(-1+B)^{3 n+1}}, y_{0}=y_{0}(-1+C)^{3 n+1}, \\
& x_{-3}=\frac{x_{-3}}{(-1+A)^{3 n+2}}, x_{-2}=x_{-2}(-1+B)^{3 n+2}, x_{-1}=\frac{x_{-1}}{(-1+C)^{3 n+2}}, x_{0}=x_{0}(-1+A)^{3 n+2},
\end{aligned}
$$

where from we get

$$
A=B=C=2 .
$$

Second, assume now that $A=B=C=2$. Then we see from the form of the solution of system (3.1) that

$$
\begin{aligned}
& x_{12 n-3}=x_{-3}, x_{12 n-2}=x_{-2}, x_{12 n-1}=x_{-1}, x_{12 n}=x_{0}, x_{12 n+1}=z_{-3}, x_{12 n+2}=z_{-2}, \\
& x_{12 n+3}=z_{-1}, x_{12 n+4}=z_{0}, x_{12 n+5}=y_{-3}, x_{12 n+6}=y_{-2}, x_{12 n+7}=y_{-1}, x_{12 n+8}=y_{0}, \\
& y_{12 n-3}=y_{-3}, y_{12 n-2}=y_{-2}, y_{12 n-1}=y_{-1}, y_{12 n}=y_{0}, y_{12 n+1}=x_{-3}, y_{12 n+2}=x_{-2}, \\
& y_{12 n+3}=x_{-1}, y_{12 n+4}=x_{0}, y_{12 n+5}=z_{-3}, y_{12 n+6}=z_{-2}, y_{12 n+7}=z_{-1}, y_{12 n+8}=z_{0}, \\
& z_{12 n-3}=z_{-3}, z_{12 n-2}=z_{-2}, z_{12 n-1}=z_{-1}, z_{12 n}=z_{0}, z_{12 n+1}=y_{-3}, z_{12 n+2}=y_{-2}, \\
& z_{12 n+3}=y_{-1}, z_{12 n+4}=y_{0}, z_{12 n+5}=x_{-3}, z_{12 n+6}=x_{-2}, z_{12 n+7}=x_{-1}, z_{12 n+8}=x_{0} .
\end{aligned}
$$

Thus we have a periodic solution of period twelve. This completes the proof.

Theorem 3.4. System (3.1) has a periodic solution of period four iff $x_{-i}=y_{-i}=z_{-i}, i=0,1,2,3, A=2$ which has the form

$$
\left\{x_{n}\right\}=\left\{y_{n}\right\}=\left\{z_{n}\right\}=\left\{x_{-3}, x_{-2}, x_{-1}, x_{0}, x_{-3}, x_{-2}, \ldots\right\} .
$$

Proof. The proof follows from the formulae of solutions of system (3.1).

Example 3.5. We consider an interesting numerical example for 3.1 with the initial conditions $x_{-3}=$ $0.6, x_{-2}=-0.3, x_{-1}=0.8, x_{0}=-1.3, y_{-3}=0.2, y_{-2}=-4, y_{-1}=0.2, y_{0}=0.7, z_{-3}=0.8, z_{-2}=$ $1.1, z_{-1}=0.02$, and $z_{0}=0.4$. See Figure 3 . 


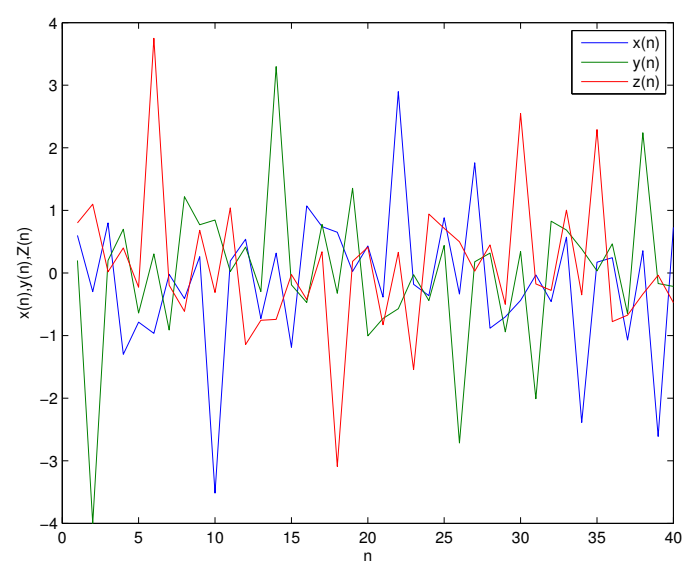

Figure 3: Plot of system 3.1.

Example 3.6. See Figure 4, when we take system (3.1) with the initial conditions $x_{-3}=6, x_{-2}=$ $1 / 14, x_{-1}=-1 / 6, x_{0}=-2, y_{-3}=0.3, y_{-2}=-3, y_{-1}=8, y_{0}=-2, z_{-3}=-7, z_{-2}=20, z_{-1}=1 / 18$, and $z_{0}=-0.5$.

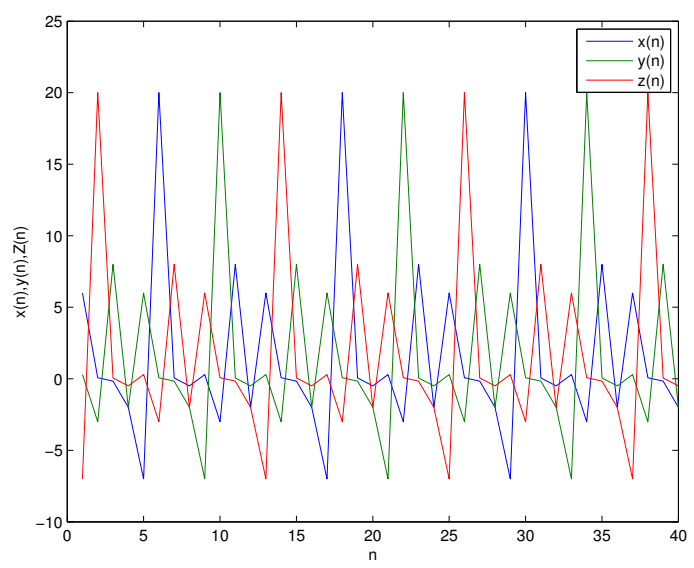

Figure 4: Plot of periodicity of system 3.1].

Example 3.7. Figure 5 shows the behavior of the solution of system (3.1) with the initial conditions $x_{-3}=y_{-3}=z_{-3}=6, x_{-2}=y_{-2}=z_{-2}=-3, x_{-1}=y_{-1}=z_{-1}=1 / 18$ and $x_{0}=y_{0}=z_{0}=-2$.

The following cases can be treated similarly.

4. The Third Case $a_{i}=b_{i}=b_{3}=1, i=1,2, a_{3}=-1$

Here we obtain a form of the solutions of the system of the difference equations

$$
\begin{aligned}
x_{n+1} & =\frac{z_{n-3}}{1+z_{n} y_{n-1} x_{n-2} z_{n-3}}, y_{n+1}=\frac{x_{n-3}}{1+x_{n} z_{n-1} y_{n-2} x_{n-3}}, \\
z_{n+1} & =\frac{y_{n-3}}{-1+y_{n} x_{n-1} z_{n-2} y_{n-3}},
\end{aligned}
$$

where $n=0,1,2, \ldots$ and the initial conditions are arbitrary real numbers with $A \neq \pm 1, \neq \frac{1}{2}, B \neq \pm 1$, $\neq-\frac{1}{2}$ and $C \neq 1, \neq \frac{1}{2}, \neq \frac{1}{3}$. 


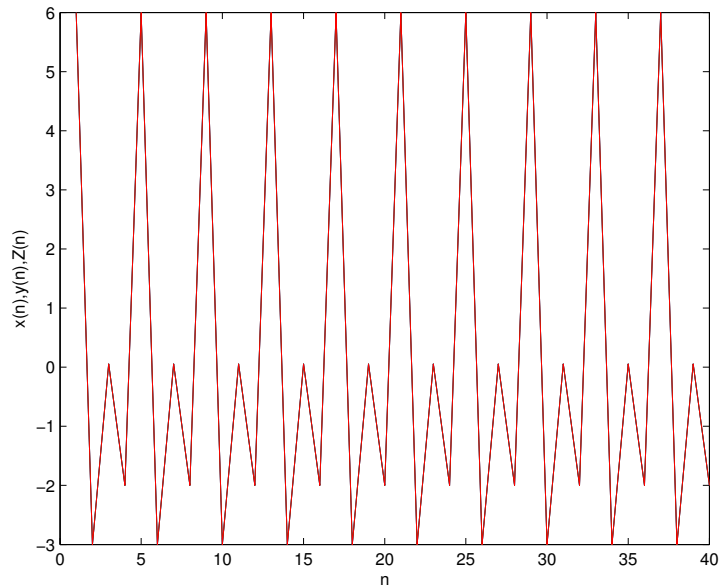

Figure 5: Plot of system (3.1) when $x_{-i}=y_{-i}=z_{-i}, i=0,1,2,3, A=2$.

Theorem 4.1. Let $\left\{x_{n}, y_{n}, z_{n}\right\}_{n=-3}^{+\infty}$ be a solution of system 4.1. Then for $n=0,1,2, \ldots$, we have

$$
\begin{aligned}
& x_{12 n-3}=\frac{x_{-3}(-1+2 A)^{n}}{(-1+A)^{2 n}(1+A)^{n}}, \\
& x_{12 n-2}=\frac{x_{-2}(-1+B)^{n}(1+B)^{2 n}}{(1+2 B)^{n}}, \\
& x_{12 n-1}=\frac{x_{-1}(1-2 C)^{2 n}}{(-1+C)^{2 n}(-1+3 C)^{n}}, \\
& x_{12 n}=\frac{x_{0}(-1+A)^{2 n}(1+A)^{n}}{(-1+2 A)^{n}}, \\
& x_{12 n+1}=\frac{z_{-3}(1+2 B)^{n}}{(-1+B)^{n}(1+B)^{2 n+1}}, \\
& x_{12 n+2}=\frac{z_{-2}(-1+C)^{2 n+1}(-1+3 C)^{n}}{(-1+2 C)^{2 n+1}}, \\
& x_{12 n+3}=-\frac{z_{-1}(-1+2 A)^{n}}{(-1+A)^{2 n+1}(1+A)^{n}}, \\
& x_{12 n+4}=\frac{z_{0}(-1+B)^{n}(1+B)^{2 n+1}}{(1+2 B)^{n}}, \\
& x_{12 n+5}=\frac{y_{-3}(-1+2 C)^{2 n+1}}{(-1+C)^{2 n+2}(-1+3 C)^{n}}, \\
& x_{12 n+6}=\frac{y_{-2}(-1+A)^{2 n+1}(1+A)^{n+1}}{(-1+2 A)^{n}} \text {, } \\
& x_{12 n+7}=-\frac{y_{-1}(1+2 B)^{n+1}}{(-1+B)^{n}(1+B)^{2 n+2}}, \\
& x_{12 n+8}=-\frac{y_{0}(-1+C)^{2 n+1}(-1+3 C)^{n+1}}{(-1+2 C)^{2 n+2}}, \\
& y_{12 n-3}=\frac{y_{-3}(-1+2 C)^{2 n}}{(-1+C)^{2 n}(-1+3 C)^{n}}, \\
& y_{12 n-2}=\frac{y_{-2}(-1+A)^{2 n}(1+A)^{n}}{(-1+2 A)^{n}}, \\
& y_{12 n-1}=\frac{y_{-1}(1+2 B)^{n}}{(-1+B)^{n}(1+B)^{2 n}}, \\
& y_{12 n}=\frac{y_{0}(-1+C)^{2 n}(-1+3 C)^{n}}{(-1+2 C)^{2 n}}, \\
& y_{12 n+1}=\frac{x_{-3}(-1+2 A)^{n}}{(-1+A)^{2 n}(1+A)^{n+1}}, \\
& y_{12 n+2}=\frac{x_{-2}(-1+B)^{n}(1+B)^{2 n+1}}{(1+2 B)^{n+1}}, \\
& y_{12 n+3}=\frac{x_{-1}(-1+2 C)^{2 n+1}}{(-1+C)^{2 n}(-1+3 C)^{n+1}}, \\
& y_{12 n+4}=\frac{x_{0}(-1+A)^{2 n+1}(1+A)^{n}}{(-1+2 A)^{n+1}}, \\
& y_{12 n+5}=-\frac{z_{-3}(1+2 B)^{n}}{(-1+B)^{n+1}(1+B)^{2 n+1}}, \\
& y_{12 n+6}=-\frac{z_{-2}(-1+C)^{2 n+2}(-1+3 C)^{n}}{(-1+2 C)^{2 n+1}}, \\
& y_{12 n+7}=-\frac{z_{-1}(-1+2 A)^{n}}{(-1+A)^{2 n+1}(1+A)^{n+1}}, \\
& y_{12 n+8}=\frac{z_{0}(-1+B)^{n}(1+B)^{2 n+2}}{(1+2 B)^{n+1}}, \\
& z_{12 n-3}=\frac{z_{-3}(1+2 B)^{n}}{(-1+B)^{n}(1+B)^{2 n}}, \\
& z_{12 n-2}=\frac{z_{-2}(-1+C)^{2 n}(-1+3 C)^{n}}{(-1+2 C)^{2 n}}, \\
& z_{12 n-1}=\frac{z_{-1}(-1+2 A)^{n}}{(-1+A)^{2 n}(1+A)^{n}}, \\
& z_{12 n}=\frac{z_{0}(-1+B)^{n}(1+B)^{2 n}}{(1+2 B)^{n}},
\end{aligned}
$$




$$
\begin{aligned}
z_{12 n+1} & =\frac{y_{-3}(-1+2 C)^{2 n}}{(-1+C)^{2 n+1}(-1+3 C)^{n}}, & z_{12 n+2} & =-\frac{y_{-2}(-1+A)^{2 n}(1+A)^{n+1}}{(-1+2 A)^{n}} \\
z_{12 n+3} & =-\frac{y_{-1}(1+2 B)^{n+1}}{(-1+B)^{n}(1+B)^{2 n+1}}, & z_{12 n+4} & =-\frac{y_{0}(-1+C)^{2 n}(-1+3 C)^{n+1}}{(-1+2 C)^{2 n+1}} \\
z_{12 n+5} & =-\frac{x_{-3}(-1+2 A)^{n+1}}{(-1+A)^{2 n+1}(1+A)^{n+1}}, & z_{12 n+6} & =\frac{x_{-2}(-1+B)^{n+1}(1+B)^{2 n+1}}{(1+2 B)^{n+1}} \\
z_{12 n+7} & =\frac{x_{-1}(-1+2 C)^{2 n+1}}{(-1+C)^{2 n+1}(-1+3 C)^{n+1}}, & z_{12 n+8} & =-\frac{x_{0}(-1+A)^{2 n+1}(1+A)^{n+1}}{(-1+2 A)^{n+1}}
\end{aligned}
$$

Example 4.2. In the following example for system (4.1), we put the initial conditions $x_{-3}=0.6, x_{-2}=$ $-0.3, x_{-1}=0.7, x_{0}=-1.3, y_{-3}=0.2, y_{-2}=-0.4, y_{-1}=0.2, y_{0}=0.7, z_{-3}=0.8, z_{-2}=0.24, z_{-1}=0.5$, and $z_{0}=0.4$. See Figure 6 .

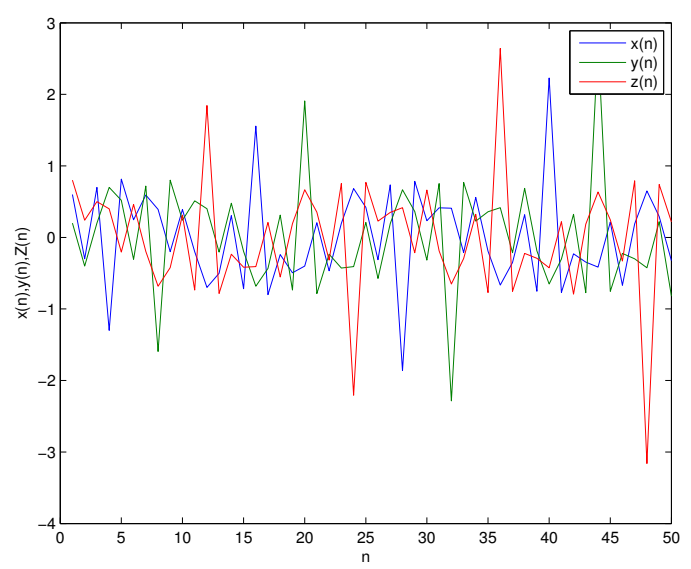

Figure 6: Plot of system 4.1.

\section{Other Cases}

In this section, we get a form of the solutions of the following systems of the difference equations

$$
\begin{aligned}
x_{n+1} & =\frac{z_{n-3}}{1+z_{n} y_{n-1} x_{n-2} z_{n-3}}, y_{n+1}=\frac{x_{n-3}}{-1-x_{n} z_{n-1} y_{n-2} x_{n-3}}, \\
z_{n+1} & =\frac{y_{n-3}}{1-y_{n} x_{n-1} z_{n-2} y_{n-3}} . \\
x_{n+1} & =\frac{z_{n-3}}{-1-z_{n} y_{n-1} x_{n-2} z_{n-3}}, y_{n+1}=\frac{x_{n-3}}{-1-x_{n} z_{n-1} y_{n-2} x_{n-3}}, \\
z_{n+1} & =\frac{y_{n-3}}{-1-y_{n} x_{n-1} z_{n-2} y_{n-3}} .
\end{aligned}
$$

Theorem 5.1. Let $\left\{x_{n}, y_{n}, z_{n}\right\}$ be a solution of system (5.1). Then for $n=0,1,2, \ldots$, we have

$$
\begin{array}{rlrl}
x_{12 n-3} & =\frac{x_{-3}(1+2 A)^{n}}{(1+A)^{2 n}(-1+A)^{n}}, & x_{12 n-2} & =\frac{x_{-2}(-1)^{n}(1+B)^{2 n}(1+3 B)^{n}}{(1+2 B)^{2 n}}, \\
x_{12 n-1} & =\frac{x_{-1}(1+2 C)^{n}}{(1+C)^{2 n}(-1+C)^{n}}, & x_{12 n}=\frac{x_{0}(1+A)^{2 n}(-1+A)^{n}}{(1+2 A)^{n}},
\end{array}
$$




$$
\begin{aligned}
& x_{12 n+1}=\frac{z_{-3}(-1)^{n}(1+2 B)^{2 n}}{(1+B)^{2 n+1}(1+3 B)^{n}}, \quad x_{12 n+2}=-\frac{z_{-2}(-1+C)^{n+1}(1+C)^{2 n}}{(1+2 C)^{n}}, \\
& x_{12 n+3}=\frac{z_{-1}(1+2 A)^{n+1}}{(1+A)^{2 n+1}(-1+A)^{n}}, \quad x_{12 n+4}=\frac{z_{0}(-1)^{n}(1+3 B)^{n+1}(1+B)^{2 n}}{(1+2 B)^{2 n+1}}, \\
& x_{12 n+5}=-\frac{y_{-3}(1+2 C)^{n+1}}{(1+C)^{2 n+1}(-1+C)^{n+1}}, \quad x_{12 n+6}=-\frac{y_{-2}(1+A)^{2 n+1}(-1+A)^{n+1}}{(1+2 A)^{n+1}}, \\
& x_{12 n+7}=\frac{y_{-1}(-1)^{n}(1+2 B)^{2 n+1}}{(1+3 B)^{n+1}(1+B)^{2 n+1}}, \quad x_{12 n+8}=-\frac{y_{0}(-1+C)^{n+1}(1+C)^{2 n+1}}{(1+2 C)^{n+1}}, \\
& y_{12 n-3}=\frac{y_{-3}(1+2 C)^{n}}{(1+C)^{2 n}(-1+C)^{n}}, \quad y_{12 n-2}=\frac{y_{-2}(1+A)^{2 n}(-1+A)^{n}}{(1+2 A)^{n}}, \\
& y_{12 n-1}=\frac{y_{-1}(-1)^{n}(1+2 B)^{2 n}}{(1+3 B)^{n}(1+B)^{2 n}}, \quad y_{12 n}=\frac{y_{0}(1+C)^{2 n}(-1+C)^{n}}{(1+2 C)^{n}}, \\
& y_{12 n+1}=-\frac{x_{-3}(1+2 A)^{n}}{(1+A)^{2 n+1}(-1+A)^{n}}, \quad y_{12 n+2}=\frac{x_{-2}(-1)^{n+1}(1+3 B)^{n}(1+B)^{2 n+1}}{(1+2 B)^{2 n+1}}, \\
& y_{12 n+3}=-\frac{x_{-1}(1+2 C)^{n}}{(1+C)^{2 n+1}(-1+C)^{n}}, \quad y_{12 n+4}=-\frac{x_{0}(1+A)^{2 n+1}(-1+A)^{n}}{(1+2 A)^{n}}, \\
& y_{12 n+5}=\frac{z_{-3}(-1)^{n+1}(1+2 B)^{2 n+1}}{(1+3 B)^{n}(1+B)^{2 n+2}}, \quad y_{12 n+6}=\frac{z_{-2}(1+C)^{2 n+1}(-1+C)^{n+1}}{(1+2 C)^{n}}, \\
& y_{12 n+7}=-\frac{z_{-1}(1+2 A)^{n+1}}{(1+A)^{2 n+2}(-1+A)^{n}}, \quad y_{12 n+8}=\frac{z_{0}(-1)^{n+1}(1+3 B)^{n+1}(1+B)^{2 n+1}}{(1+2 B)^{2 n+2}}, \\
& z_{12 n-3}=\frac{z_{-3}(-1)^{n}(1+2 B)^{2 n}}{(1+3 B)^{n}(1+B)^{2 n}}, \quad z_{12 n-2}=\frac{z_{-2}(1+C)^{2 n}(-1+C)^{n}}{(1+2 C)^{n}}, \\
& z_{12 n-1}=\frac{z_{-1}(1+2 A)^{n}}{(1+A)^{2 n}(-1+A)^{n}}, \quad z_{12 n}=\frac{z_{0}(-1)^{n}(1+3 B)^{n}(1+B)^{2 n}}{(1+2 B)^{2 n}}, \\
& z_{12 n+1}=-\frac{y_{-3}(1+2 C)^{n}}{(-1+C)^{n+1}(1+C)^{2 n}}, \quad z_{12 n+2}=\frac{y_{-2}(-1+A)^{n}(1+A)^{2 n+1}}{(1+2 A)^{n+1}}, \\
& z_{12 n+3}=\frac{y_{-1}(-1)^{n}(1+2 B)^{2 n+1}}{(1+B)^{2 n}(1+3 B)^{n+1}}, \quad z_{12 n+4}=\frac{y_{0}(1+C)^{2 n+1}(-1+C)^{n}}{(1+2 C)^{n+1}}, \\
& z_{12 n+5}=\frac{x_{-3}(1+2 A)^{n}}{(1+A)^{2 n+1}(-1+A)^{n+1}}, \quad z_{12 n+6}=\frac{x_{-2}(-1)^{n+1}(1+3 B)^{n}(1+B)^{2 n+2}}{(1+2 B)^{2 n+1}}, \\
& z_{12 n+7}=\frac{x_{-1}(1+2 C)^{n}}{(1+C)^{2 n+1}(-1+C)^{n+1}}, \quad z_{12 n+8}=-\frac{x_{0}(1+A)^{2 n+2}(-1+A)^{n}}{(1+2 A)^{n+1}},
\end{aligned}
$$

where $A, C \neq \pm 1, \neq-\frac{1}{2}$ and $B_{0} \neq-1, \neq-\frac{1}{2}, \neq-\frac{1}{3}$.

Theorem 5.2. Let $\left\{x_{n}, y_{n}, z_{n}\right\}_{n=-3}^{+\infty}$ be a solution of system 5.2 and $A, B, C \neq-1$. Then for $n=$ $0,1,2, \ldots$, we have

$$
\begin{aligned}
x_{12 n-3} & =\frac{x_{-3}}{(-1-A)^{3 n}}, x_{12 n-2}=x_{-2}(-1-B)^{3 n}, x_{12 n-1}=\frac{x_{-1}}{(-1-C)^{3 n}}, \\
x_{12 n} & =x_{0}(-1-A)^{3 n}, x_{12 n+1}=\frac{z_{-3}}{(-1-B)^{3 n+1}}, x_{12 n+2}=z_{-2}(-1-C)^{3 n+1}, \\
x_{12 n+3} & =\frac{z_{-1}}{(-1-A)^{3 n+1}}, x_{12 n+4}=z_{0}(-1-B)^{3 n+1}, x_{12 n+5}=\frac{y_{-3}}{(-1-C)^{3 n+2}}, \\
x_{12 n+6} & =y_{-2}(-1-A)^{3 n+2}, x_{12 n+7}=\frac{y_{-1}}{(-1-B)^{3 n+2}}, x_{12 n+8}=y_{0}(-1-C)^{3 n+2}, \\
y_{12 n-3} & =\frac{y_{-3}}{(-1-C)^{3 n}}, \quad y_{12 n-2}=y_{-2}(-1-A)^{3 n}, y_{12 n-1}=\frac{y_{-1}}{(-1-B)^{3 n}},
\end{aligned}
$$




$$
\begin{aligned}
y_{12 n} & =y_{0}(-1-C)^{3 n}, y_{12 n+1}=\frac{x_{-3}}{(-1-A)^{3 n+1}}, y_{12 n+2}=x_{-2}(-1-B)^{3 n+1}, \\
y_{12 n+3} & =\frac{x_{-1}}{(-1-C)^{3 n+1}}, y_{12 n+4}=x_{0}(-1-A)^{3 n+1}, y_{12 n+5}=\frac{z_{-3}}{(-1-B)^{3 n+2}}, \\
y_{12 n+6} & =z_{-2}(-1-C)^{3 n+2}, y_{12 n+7}=\frac{z_{-1}}{(-1-A)^{3 n+2}}, y_{12 n+8}=z_{0}(-1-B)^{3 n+2}, \\
z_{12 n-3} & =\frac{z_{-3}}{(-1-B)^{3 n}}, z_{12 n-2}=z_{-2}(-1-C)^{3 n}, z_{12 n-1}=\frac{z_{-1}}{(-1-A)^{3 n}}, \\
z_{12 n} & =z_{0}(-1-B)^{3 n}, z_{12 n+1}=\frac{y_{-3}}{(-1-C)^{3 n+1}}, z_{12 n+2}=y_{-2}(-1-A)^{3 n+1}, \\
z_{12 n+3} & =\frac{y_{-1}}{(-1-B)^{3 n+1}}, z_{12 n+4}=y_{0}(-1-C)^{3 n+1}, z_{12 n+5}=\frac{x_{-3}}{(-1-A)^{3 n+2}}, \\
z_{12 n+6} & =x_{-2}(-1-B)^{3 n+2}, z_{12 n+7}=\frac{x_{-1}}{(-1-C)^{3 n+2}}, z_{12 n+8}=x_{0}(-1-A)^{3 n+2} .
\end{aligned}
$$

Lemma 5.3. The solutions of system (5.2) are unbounded except in the cases given in the following two theorems.

Theorem 5.4. System (5.2) has a periodic solution of period twelve iff $A=B=C=-2$ and it has the form

$$
\begin{aligned}
& \left\{x_{n}\right\}=\left\{x_{-3}, x_{-2}, x_{-1}, x_{0}, z_{-3}, z_{-2}, z_{-1}, z_{0}, y_{-3}, y_{-2}, y_{-1}, y_{0}, x_{-3}, x_{-2}, x_{-1}, x_{0}, \ldots\right\}, \\
& \left\{y_{n}\right\}=\left\{y_{-3}, y_{-2}, y_{-1}, y_{0}, x_{-3}, x_{-2}, x_{-1}, x_{0}, z_{-3}, z_{-2}, z_{-1}, z_{0}, y_{-3}, y_{-2}, y_{-1}, y_{0}, \ldots\right\}, \\
& \left\{z_{n}\right\}=\left\{z_{-3}, z_{-2}, z_{-1}, z_{0}, y_{-3}, y_{-2}, y_{-1}, y_{0}, x_{-3}, x_{-2}, x_{-1}, x_{0}, z_{-3}, z_{-2}, z_{-1}, z_{0}, \ldots\right\} .
\end{aligned}
$$

Theorem 5.5. System (5.2) has a periodic solution of period four iff $x_{-i}=y_{-i}=z_{-i}, i=0,1,2,3, A=-2$ and it is of the form

$$
\left\{x_{n}\right\}=\left\{y_{n}\right\}=\left\{z_{n}\right\}=\left\{x_{-3}, x_{-2}, x_{-1}, x_{0}, x_{-3}, x_{-2}, \ldots\right\} .
$$

\section{Acknowledgements}

The author thanks the editor and the referees for their constructive comments and suggestions which improved the quality of the paper.

\section{References}

[1] R. P. Agarwal, Difference Equations and Inequalities, $1^{\text {st }}$ edition, Marcel Dekker, New York, (1992), $2^{\text {nd }}$ edition, (2000).1

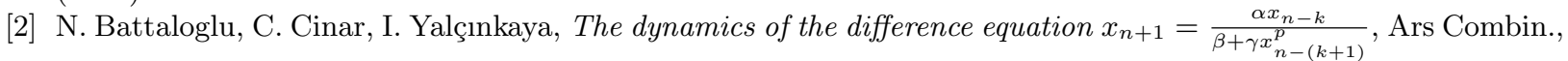
97 (2010), 281-288.

[3] M. B. Bekker, M. J. Bohner, H. D. Voulov, Asymptotic behavior of solutions of a rational system of difference equations, J. Nonlinear Sci. Appl., 7 (2014), 379-382.

[4] E. Camouzis, M. R. S. Kulenović, G. Ladas, O. Merino, Rational systems in the plane, J. Difference Equ. Appl., 15 (2009), 303-323.

[5] C. Cinar, On the positive solutions of the difference equation system $x_{n+1}=\frac{1}{y_{n}}, y_{n+1}=\frac{y_{n}}{x_{n-1} y_{n-1}}$, Appl. Math. Comput., 158 (2004), 303-305.1

[6] D. Clark, M. R. S. Kulenović, A coupled system of rational difference equations, Comput. Math. Appl., 43 (2002), 849-867.1

[7] D. Clark, M. R. S. Kulenović, J. F. Selgrade, Global asymptotic behavior of a two-dimensional difference equation modelling competition, Nonlinear Anal., 52 (2003), 1765-1776.1

[8] S. E. Das, M. Bayram, On a system of rational difference equations, World App. Sci. J., 10 (2010), 1306-1312.

[9] Q. Din, M. N. Qureshi, A. Qadeer Khan, Dynamics of a fourth-order system of rational difference equations, Adv. Difference Equ., 2012 (2012), 15 pages.1

[10] E. M. Elabbasy, S. M. Eleissawy, Asymptotic behavior of two dimensional rational system of difference equations, Dyn. Contin. Discrete Impuls. Sys. Ser. B Appl. Algorithms, 20 (2013), 221-235.

[11] S. Elaydi, An Introduction to Difference Equations, Undergrad. Texts Math., Springer, New York, NY, USA, $3^{\text {rd }}$ edition, (2005). 
[12] M. M. El-Dessoky, On a solvable for some nonlinear systems of difference equations, J. Comput. Theor. Nanosci., 12 (2015), 3432-3442.

[13] M. M. El-Dessoky, E. M. Elsayed, On a solution of system of three fractional difference equations, J. Comp. Anal. Appl., 19 (2015), 760-769.

[14] M. M. El-Dessoky, M. Mansour, E. M. Elsayed, Solutions of some rational systems of difference equations, Util. Math., 92 (2013), 329-336.1

[15] E. M. Elsayed, Solutions of rational difference system of order two, Math. Comput. Model., 55 (2012), 378-384.

[16] E. M. Elsayed, M. M. El-Dessoky, A. Alotaibi, On the solutions of a general system of difference equations, Discrete Dyn. Nat. Soc., 2012 (2012), 12 pages.

[17] E. M. Elsayed, M. M. El-Dessoky, E. O. Alzahrani, The form of the solution and dynamic of a rational recursive sequence, J. Comput. Anal. Appl., 17 (2014), 172-186.

[18] E. M. Elsayed, M. Mansour, M. M. El-Dessoky, Solutions of fractional systems of difference equations, Ars Combin., 110 (2013), 469-479.

[19] M. E. Erdoğan, C. Cinar, I. Yalçınkaya, On the dynamics of the recursive sequence, Comput. Math. Appl., 61 (2011), 533-537.

[20] E. A. Grove, G. Ladas, L. C. McGrath, C. T. Teixeira, Existence and behavior of solutions of a rational system, Commun. Appl. Nonlinear Anal., 8 (2001), 1-25.

[21] V. L. Kocić, G. Ladas, Global Behavior of Nonlinear Difference Equations of Higher Order with Applications, Kluwer, Dordrecht, (1993).

[22] A. S. Kurbanli, On the behavior of solutions of the system of rational difference equations: $x_{n+1}=$ $\frac{x_{n-1}}{x_{n-1} y_{n}-1}, y_{n+1}=\frac{y_{n-1}}{y_{n-1} x_{n}-1}$, and $z_{n+1}=\frac{z_{n-1}}{z_{n-1} y_{n}-}$, Discrete Dyn. Nat. Soc., 2011 (2011), 12 pages.

[23] A. S. Kurbanli, On the behavior of solutions of the system of rational difference equations, Adv. Differ. Equ., 2011 (2011), 8 pages.

[24] A. S. Kurbanli, C. Cinar, I. Yalçınkaya, On the behavior of positive solutions of the system of rational difference equations $x_{n+1}=\frac{x_{n-1}}{y_{n} x_{n-1}+1}, y_{n+1}=\frac{y_{n-1}}{x_{n} y_{n-1}+1}$, Math. Comput. Model., 53 (2011), 1261-1267.1

[25] K. Liu, Z. Wei, P. Li, W. Zhong, On the behavior of a system of rational difference equations $x_{n+1}=\frac{x_{n-1}}{x_{n-1} y_{n}-1}$, $y_{n+1}=\frac{y_{n-1}}{y_{n-1} x_{n}-1}, z_{n+1}=\frac{1}{z_{n-1} x_{n}}$, Discrete Dyn. Nat. Soc., 2012 (2012), 9 pages. 1

[26] H. Ma, H. Feng, On positive solutions for the rational difference equation systems $x_{n+1}=\frac{A}{x_{n} y_{n}^{2}}, y_{n+1}=$ $\frac{B y_{n}}{x_{n-1} y_{n-1}}$, Int. Schol. Res. Notices, 2014 (2014), 4 pages.1

[27] M. Mansour, M. M. El-Dessoky, E. M. Elsayed, On the solution of rational systems of difference equations, J. Comp. Anal. Appl., 15 (2013), 967-976.

[28] A. Y. Ozban, On the positive solutions of the system of rational difference equations $x_{n+1}=\frac{1}{y_{n-k}}, y_{n+1}=$ $\frac{y_{n}}{x_{n-m} y_{n-m-k}}$, J. Math. Anal. Appl., 323 (2006), 26-32.1

[29] G. Papaschinopoulos, G. Ellina, K. B. Papadopoulos, Asymptotic behavior of the positive solutions of an exponential type system of difference equations, Appl. Math. Comput., 245 (2014), 181-190.1

[30] B. Sroysang, Dynamics of a system of rational higher-order difference equation, Discrete Dyn. Nat. Soc., 2013 (2013), 5 pages. 1

[31] S. Stević, On a solvable system of difference equations of fourth order, Appl. Math. Comput., 219 (2013), 57065716.

[32] N. Touafek, On some fractional systems of difference equations, Iran. J. Math. Sci. Inform., 9 (2014), 73-86.

[33] N. Touafek, E. M. Elsayed, On the solutions of systems of rational difference equations, Math. Comput. Model., 55 (2012), 1987-1997.1

[34] H. Yacine, Form and periodicity of solutions of some systems of higher-order difference equations, Math. Sci. Lett., 5 (2016), 79-84.

[35] I. Yalcinkaya, C. Cinar, M. Atalay, On the solutions of systems of difference equations, Adv. Difference Equ., 2008 (2008), 9 pages.

[36] X. Yang, Y. Liu, S. Bai, On the system of high order rational difference equations $x_{n}=\frac{a}{y_{n-p}}, y_{n}=\frac{b y_{n-p}}{x_{n-q} y_{n-q}}$, Appl. Math. Comput., 171 (2005), 853-856.

[37] Q. Zhang, J. Liu, Z. Luo, Dynamical behavior of a system of third-order rational difference equation, Discrete Dyn. Nat. Soc., 2015 (2015), 6 pages.11

[38] Q. Zhang, W. Zhang, Y. Shao, J. Liu, On the system of high order rational difference equations, Int. Schol. Res. Notices, 2014 (2014), 5 pages.

[39] O. Zkan, A. S. Kurbanli, On a system of difference equation, Discrete Dyn. Nat. Soc., 2013 (2013), 7 pages.1 